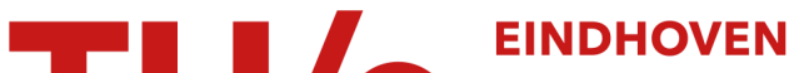 UNIVERSITY OF TECHNOLOGY
}

\section{Harmonic disturbance location by applying Bayesian inference}

Citation for published version (APA):

Ye, G., Xiang, Y., Cuk, V., \& Cobben, J. F. G. (2016). Harmonic disturbance location by applying Bayesian inference. Electric Power Systems Research, 140, 886-894. https://doi.org/10.1016/j.epsr.2016.04.016

DOI:

10.1016/j.epsr.2016.04.016

Document status and date:

Published: 01/01/2016

\section{Document Version:}

Accepted manuscript including changes made at the peer-review stage

\section{Please check the document version of this publication:}

-A submitted manuscript is the version of the article upon submission and before peer-review. There can be important differences between the submitted version and the official published version of record. People interested in the research are advised to contact the author for the final version of the publication, or visit the $\mathrm{DOI}$ to the publisher's website.

- The final author version and the galley proof are versions of the publication after peer review.

- The final published version features the final layout of the paper including the volume, issue and page numbers.

Link to publication

\section{General rights}

Copyright and moral rights for the publications made accessible in the public portal are retained by the authors and/or other copyright owners and it is a condition of accessing publications that users recognise and abide by the legal requirements associated with these rights.

- Users may download and print one copy of any publication from the public portal for the purpose of private study or research.

- You may not further distribute the material or use it for any profit-making activity or commercial gain

- You may freely distribute the URL identifying the publication in the public portal.

If the publication is distributed under the terms of Article 25fa of the Dutch Copyright Act, indicated by the "Taverne" license above, please follow below link for the End User Agreement:

www.tue.nl/taverne

Take down policy

If you believe that this document breaches copyright please contact us at:

openaccess@tue.nl

providing details and we will investigate your claim. 


\title{
Harmonic Disturbance Location by Applying Bayesian Inference
}

\author{
G.Ye*,Y.Xiang,V.Cuk, and J.F.G.Cobben \\ Technology University of Eindhoven, The Netherlands \\ g.ye@tue.nl \\ Roomnr. 2.076, Gebouw 19 Flux, De Groene Loper, 5600MB, Eindhoven, The Netherlands
}

\begin{abstract}
Harmonic pollution is one of the most important power quality issues in electric power systems. Correct location of the main harmonic disturbance source is a key step to solve the problem. This paper presents a method to detect the location of harmonic disturbance source in low voltage network through Bayesian inference. The harmonic state is estimated based on the measurement data from limited measurement points whereby the measurement error is also considered. The performance of the proposed method is assessed through a case study applied in a typical low voltage network. Monte Carlo simulation is used to obtain the statistical results. The influence of different parameters like disturbance level and measurement accuracy level etc. are discussed. The proposed method shows the adequacy for the analysis of distribution networks.
\end{abstract}

\section{Introduction}

One of the significant changes in current distribution network is the large applications of power electronic devices due to the rapid development of semiconductor technology. Therefore more and more nonlinear loads are connected to the system which results in higher harmonic pollution levels. According to [1], the survey conducted by the Electric Power Research Institute shows that only 15\%-20\% of the total loads were nonlinear in 1992 while the percentage of nonlinear loads will be over $50 \%$ of all loads in the future. Harmonic pollution may have serious consequences to the system such as overheating and damaging of equipment, unexpected tripping of sensitive loads, failure of protective relays, and disturbance to the communication circuit.

Locating the main harmonic disturbance source is important to improve the power quality (PQ) level of the network. Moreover, it is crucial for the distribution system operator (DSO) to share the responsibilities. There are many literatures discussing the identification and detection of harmonic sources in distribution systems. In [2], the vector-matrix form the Kirchhoff law is solved with a hybrid weighted least squares estimator, and then the harmonic signal magnitudes are obtained. The harmonic power direction-based method is one of the most common tools to figure out the solution [3], [4]. The principle of the method is to investigate the direction of harmonic active power. If it is from the utility to customer, the utility is considered to be dominant to 
the pollution, and vice versa. The harmonic power approach is improved according to [5] in which several concerns are addressed for the application to radial system and a modification is proposed to generalize its application to non-radial systems. Simulations and experiments carried out in [6] present that the single-point strategy can give useful indications for the detection of the dominant harmonic source, upstream or downstream the measurement point. The strategy is improved in [7] which has been proved to be successful in several simulations. In [1], Saxena et al. proposed a method based on the direction of harmonic power flow at system nodes with the measurement of voltage phasor measuring unit, which are used to categorize and rank the suspected buses. In [8], sensitivity analysis and the minimum variance criterion are utilized to determine the optimal locations of measurement devices for locating harmonic sources. It is proposed in [9], [10], [11], [12] to calculate the harmonic sources of the utility and customer with the measured harmonic impedances at point of connection (PoC). The Norton model is applied to represent the harmonic equivalent circuit. In [13], Ma and Girgis addressed two problems: the optimal locations of a limited number of harmonic meters and the optimal dynamic estimation of harmonic source locations, and it is solved by the Kalman-filtering-based technique. The principle of critical impedance is applied to identify the harmonic disturbance according to [14], [15]. The exact information of the internal impedances or admittance both of the supplier and customer sides' equivalent circuits is demanded for this method. In [16], a combined approach of superposition and critical impedance is reported which is considered as an improvement of critical impedance method. In [17], Dan proposed a method of monoparameter variation for the identification of the existence of a harmonic source. Harmonic state estimation (HSE) which is capable to calculate the harmonic generation and penetration throughout the grid is applied to locate the nonlinear load in the network [18], [19], [20]. Modern techniques are also applied to harmonic characterization and detection. The neural network algorithm is applied in [21], [22], [23], the fuzzy logic is utilized in [24], the method based on independent component analysis and mutual information theory [25] and Yang et al. introduced a data clustering method in [26].

This paper proposes a harmonic disturbance location (HDL) approach based on Bayesian inference which takes the measurement errors and load diversities into account. Bayesian inference is a method of inference in which Bayes' theorem is applied to calculate the probability distribution. The inference applies existing information (based on expert knowledge, past studies, etc.) into the data analysis. This existing information is represented as a prior distribution, and the data likelihood is effectively weighted by the prior distribution when the data analysis results are calculated. Statistical results can be obtained which are the rankings of suspected loads. In section II, the mathematical model behind the problem and the location method framework are presented. In section III, the prior distribution of the harmonic background distortion and injection is calculated. Section IV defines the grid condition and the measurement framework. In section $\mathrm{V}$, the solution of the posterior distribution of the harmonic disturbance source is given. Section VI addresses on the numerical concerns for the high-dimensional integral. In section VII, the detailed parameters of the network mod- 


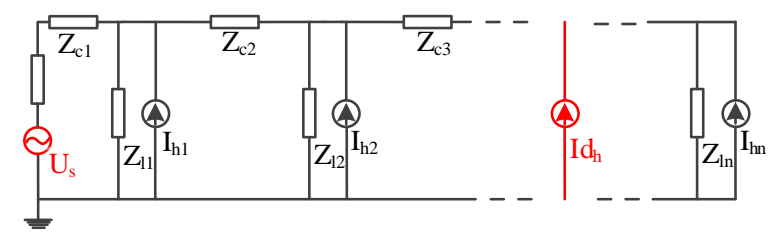

Figure 1: The equivalent circuit for harmonic disturbance location

elling and the simulation algorithm for the case study are presented. In section VIII, several scenarios are carried out to investigate the impacts of different factors, like levels of harmonic disturbance, the number and locations of measurement devices, measurement parameters and measurement accuracy levels as well as the definition of the prior distribution. Comparing with other methods, the advantages of the proposed method are discussed.

\section{Harmonic Disturbance Problem and its Location Framework}

\subsection{Harmonic Disturbance Problem}

Distribution grids are mostly operated in radial structure. The power is delivered to the customers connected along the feeder. Fig. 1 shows the equivalent circuit for harmonic disturbance location. The harmonic characteristic of each load is represented by the Norton equivalent model and the grid side is represented by the Thevenin equivalent model. The high level of harmonic pollution may be caused by the grid voltage $\left(U_{s}\right)$ or a disturbing customer which produces extremely high level of harmonic current $\left(I_{d h}\right)$ when the other customers' injections are under certain values $\left(I_{h 1}\right.$ to $\left.I_{h n}\right)$. $Z_{c 1}$ to $Z_{c n}$ represent the cable parameters. $Z_{l 1}$ to $Z_{l n}$ give the load parameters. If one of these customers deteriorates the power quality, it is necessary to analyse the disturbance for the responsibility-sharing and problem-solving.

\subsection{Harmonic Disturbance Location Framework}

Fig. 2 shows the methodology framework, which illustrates the proposed HDL approach. It includes 3 parts; 1 ) the pre-processing to obtain the prior distribution of harmonic background distortion and injection, 2) the Bayesian inference to acquire the posterior distribution, and 3) the ranking of possible harmonic disturbance locations.

In the pre-processing part, the prior distribution (marginal distribution) of harmonic background distortion and injection is acquired based on several groups of input data. The field measurement database and historical harmonic disturbance statistics are used to determine the harmonic injection distribution. The grid condition for the Bayesian inference is obtained through state estimation [27]. The measured voltages and currents will also be recorded and provided to the inference process. With the network parameters, the grid condition and measured quantities, the posterior distribution (conditional distribution) can be obtained with the application of Bayesian theorem. The network parameters give the information of components (cable, transformer) parameters, the grid impedance, the number of customers, and the length of cable sections between 


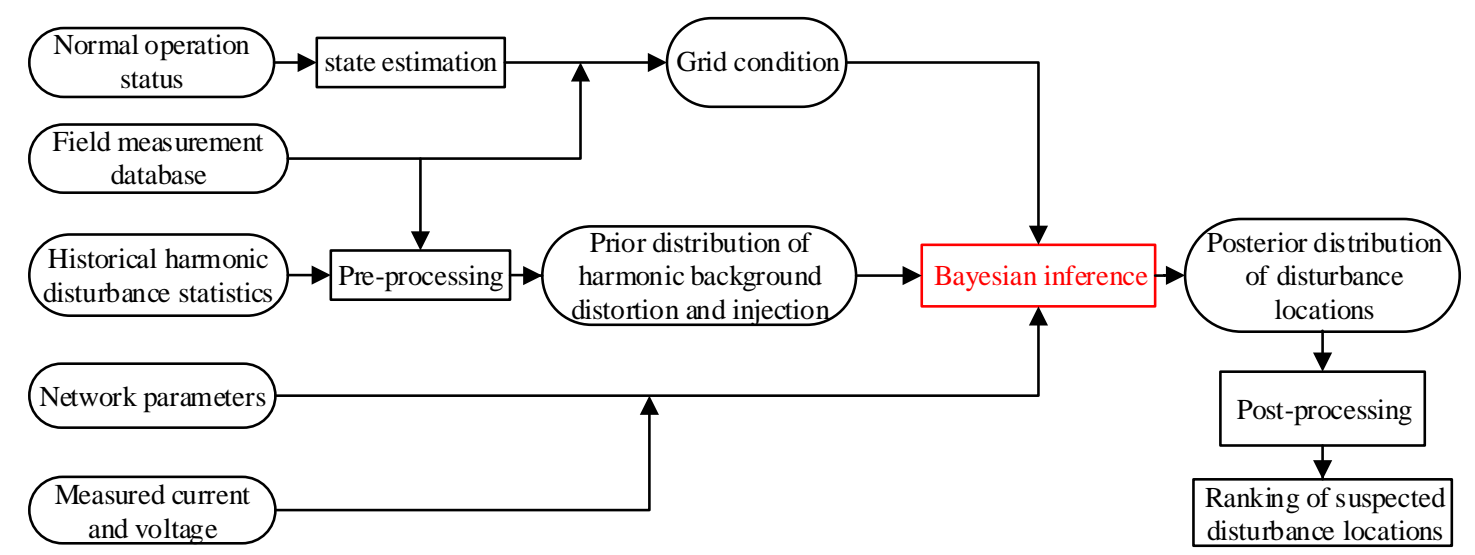

Figure 2: The framework of harmonic disturbance location

each pair of customers. In the post-processing part, the probability that the main harmonic disturbance is from the background distortion or each customer is calculated. Eventually, the ranking of possible harmonic disturbance location is acquired.

Bayesian inference is applied to detect the exact location of the disturbance source and the Bayes formula is given in (1) [28]. Bayesian probability models compute a conditional probability based on new data information and former probabilistic beliefs. The model parameters in Bayesian inference are usually treated as random rather than fixed quantities which are applied in classical statistics.

$$
f_{\boldsymbol{\Theta}}\left(\boldsymbol{\Theta} \mid x_{1}, \cdots, x_{n}\right)=\frac{f_{\boldsymbol{x}}\left(x_{1}, \cdots, x_{n}, \mid \boldsymbol{\Theta}\right) f_{\boldsymbol{\Theta}}(\boldsymbol{\Theta})}{\int_{\Omega_{\boldsymbol{\Theta}}} f_{\boldsymbol{x}}\left(x_{1}, \cdots, x_{n}, \mid \boldsymbol{\Theta}\right) f_{\boldsymbol{\Theta}}(\boldsymbol{\Theta}) d \boldsymbol{\Theta}}
$$

where $\Theta$ is considered as a continuous (similar for discrete or mixed random variables) random variable (a scalar or vector) which needs to be investigated. $f_{\Theta}(\Theta)$ is the probability density function (PDF) of the prior distribution. $f_{\boldsymbol{x}}(\boldsymbol{x} \mid \boldsymbol{\Theta})$ is the conditional pdf of the observation $\boldsymbol{x}$ which is called the Bayesian likelihood function where $\boldsymbol{x}=\left(x_{1}, \cdots, x_{n}\right)$ is the observed data vector. $f_{\boldsymbol{\Theta}}(\boldsymbol{\Theta} \mid \boldsymbol{x})$ is called posterior pdf and it is the central object of interest. $\Omega_{\Theta}$ is the whole space of $\Theta$ where the integral is calculated. The pdf of $\boldsymbol{\Theta}$ is assumed and existed beforehand, the observation of data $\boldsymbol{x}$ will affect the information of $\boldsymbol{\Theta}$ and the Bayes' rule is used to update the information further. The posterior pdf thus conveys the knowledge of $\Theta$ with the consideration of the vector $\boldsymbol{x}$ [29]. In other words, the posterior $f_{\Theta}(\boldsymbol{\Theta} \mid \boldsymbol{x})$ depends on the converse likelihood $f_{\boldsymbol{x}}(\boldsymbol{x} \mid \boldsymbol{\Theta})$ and the prior pdf $f_{\boldsymbol{\Theta}}(\boldsymbol{\Theta})$. The pdf $f_{\boldsymbol{\Theta}}(\boldsymbol{\Theta})$ determines the scale of pdf $f_{\boldsymbol{x}}(\boldsymbol{x} \mid \Theta)$, which means it can definitely affect the accuracy of the posterior pdf $f_{\boldsymbol{\Theta}}(\boldsymbol{\Theta} \mid \boldsymbol{x})$. According to the Bayesian statistics, the prior distribution can come from the existed knowledge, besides, it can also come from the objective data or statistical hypothesis tests like chi-squared or Kolmogorov-Smirnov tests [30]. In this paper, $\Theta$ represents the harmonic disturbance location and $\boldsymbol{x}$ is the measurement data. $f_{\Theta}(\boldsymbol{\Theta} \mid \boldsymbol{x})$ gives the ranking of suspected disturbance locations and is calculated with the priori $f_{\boldsymbol{\Theta}}(\boldsymbol{\Theta})$ and measurement data $\boldsymbol{x}$. 


\section{Prior Distribution of Harmonic Background Distortion and Injection}

The prior distribution which refers to the marginal distribution needs to be determined based on the fact that the harmonic disturbance can be either from the background voltage (grid side) or the customer harmonic injection (load side). The probability of the harmonic disturbance at the grid side (which means the harmonic disturbance is originated from the whole external grid) is defined as $P_{G D}$, and the probability of the harmonic disturbance at load side is defined as $P_{L D}$.

$$
P_{G D}=P_{g}, \quad P_{L D}=1-P_{G D}
$$

where $P_{g}$ is the basic probability of harmonic disturbance at grid side.

Therefore, the potential harmonic disturbance location $L$ is a discrete random variable represented by a probability mass function $(\mathrm{PMF})$, which are $f_{L, D}(l)$. In Fig. 2 , the prior distribution is obtained through a pre-processing with field measurement database and historical harmonic disturbance statistics. For the sake of simplicity, the basic prior distribution is assumed to be uniform in this paper. So $P_{g}$ is $1 /\left(1+N_{\text {load }}\right)$ where $N_{\text {load }}$ is the number of customers.

$$
f_{L, G D}(l)= \begin{cases}P_{G D} & l \in S_{G D} \\ P_{L D} / N_{\text {load }} & l \in S_{L D}\end{cases}
$$

where $S_{G D}$ represents the grid side and $S_{L D}$ is the set of all customers which can be shown as

$$
\begin{cases}S_{G D}=\{0\} & \text { grid side } \\ S_{L D}=\left\{1,2, \cdots N_{\text {load }}\right\} & \text { load side }\end{cases}
$$

where the 0 means the disturbance is from grid side and the 1 to $N_{\text {load }}$ means one particular customer originates the disturbance.

\section{Grid Condition and Measurement}

To carry out the Bayesian inference, grid condition needs to be defined and voltage and current signals needs to be measured.

\subsection{Grid Condition}

The grid condition is a vector that gives all the input parameters of the inference except the disturbance location. The parameters vector $\boldsymbol{G}_{c}$ is considered as random variables from different distributions to consider the uncertainties. In this paper, $\boldsymbol{G}_{c}$ is given as

$$
\boldsymbol{G}_{c}=\left[Z_{g}, \boldsymbol{S}_{\text {load }}, U_{\text {sh }}, \boldsymbol{I}_{\text {hload }}\right]
$$

where $Z_{g}$ is the impedance of the grid side. $\boldsymbol{S}_{\text {load }}$ is the vector of the loads in the network. $U_{s h}$ refers to the background harmonic distortion and $\boldsymbol{I}_{\text {hload }}$ represents the injected $h$-order harmonic currents of loads. 
$Z_{g}$ is the impedance of the grid side in front of the first measurement point, which is at the secondary busbar of the transformer. So it includes the external grid impedance $Z_{e g}$ (the impedance of higher voltage levels) and the transformer impedance $Z_{t}$. It is difficult to estimate $Z_{e g}$ accurately, so a normal distribution is applied to define $Z_{e g}$.

$$
Z_{e g} \sim \mathcal{N}\left(Z_{e g, \mu}, Z_{e g, \delta}^{2}\right)
$$

where $Z_{e g, \mu}$ is the mean value of the normal distribution for $Z_{e g}$ and $Z_{e g, \delta}$ is the assumed standard deviation. The values of short-circuit power at medium voltage (MV) side of the $\mathrm{MV} / \mathrm{LV}$ transformers are mostly between 50MVA and 150MVA and the value of $X / R$ is about 0.5 for a cable network [31]. $Z_{e g, \mu}$ can be calculated with these parameters.

$\boldsymbol{S}_{\text {load }}$ is the vector of the loads in the network, can be shown as

$$
\boldsymbol{S}_{\text {load }}=\left[\begin{array}{lll}
P l_{i} & P f_{i}
\end{array}\right] \quad 1 \leqslant i \leqslant N_{\text {load }}
$$

where $P l_{i}$ is the active power of the $i_{t h}$ load and $P f_{i}$ is the power factor of $i_{t h}$ load. $\boldsymbol{S}_{\text {load }}$ is calculated via state estimation and the applied approach is illustrated in [27]. Given the estimated load status, the real load status is assumed to follow the multivariate normal distribution with mean $\boldsymbol{S}_{l o a d, \mu}$ and covariance matrix $\Sigma_{s e}$. The covariance matrix $\Sigma_{s e}$ represents the state estimation error and it is a diagonal matrix containing the variance of all the individual components: $P l_{\delta, i}$ and $P f_{\delta, i}$.

$U_{s h}$ represents the background harmonic distortion. Field measurements of LV and MV networks were carried out in several European countries and the voltage distortion results are given in [31], [32], [33]. These measurement data are used as the basis to determine the values of the parameters for $\boldsymbol{G}_{c}$. In this paper, the background voltage distortion is assumed as a uniform distribution during the measurement period.

$$
U_{s h} \sim \mathcal{U}\left(U_{s h, p \min }, U_{s h, p \max }\right) \cdot U_{n}
$$

where $U_{s h, p_{\min }}$ and $U_{s h, p_{\max }}$ are the low and high levels of the magnitudes for harmonic current in percentage of nominal voltage $U_{n} . U_{s h, d p_{\min }}$ and $U_{s h, d p_{\max }}$ are the limits applied when the grid is the harmonic source.

$\boldsymbol{I}_{\text {hload }}$ is the vector of the injected h-order harmonic current of each load which can be shown as

$$
\boldsymbol{I}_{\text {hload }}=\left[I_{h, p, i} \Theta_{\text {hload }, i}\right] \quad 1 \leqslant i \leqslant N_{\text {load }}
$$

where $I_{h, p, i}$ is the magnitude for $\boldsymbol{I}_{\text {hload }}$ of the $i t h$ load and $\Theta_{\text {hload,i }}$ represents the phase angle for $\boldsymbol{I}_{\text {hload }}$ of the ith load. The uniform distribution is applied for the values which is a function of fundamental current.

$$
I_{h, p, i} \sim \mathcal{U}\left(I_{h, p \min }, I_{h, p \max }\right) \cdot I_{f, i} \quad 1 \leqslant i \leqslant N_{\text {load }}
$$

where $I_{h, p \min }$ and $I_{h, p \max }$ are the low and high levels of the magnitudes for harmonic current in percentage of the fundamental current magnitude $I_{f, i}$. These limits are 
for non-disturbing customers. In [31], different household devices are connected to define different types of household loads, which are called low, average, high load cases respectively. The harmonic spectrum of these models is measured up to 50 th harmonic order which can be applied to define the distribution of $I_{h, p, i}$. $I_{h, p, i}$ also includes the disturbing customer, $I_{d h, p \min }$ and $I_{d h, p \max }$ are the low and high level for disturbance, which are much higher than $I_{h, p \min }$ and $I_{h, p \max }$.

$$
\Theta_{\text {hload }, i} \sim \mathcal{U}\left(\Theta_{h, \min }, \Theta_{h, \max }\right) \quad 1 \leqslant i \leqslant N_{\text {load }}
$$

where $\Theta_{h \text {,min }}$ and $\Theta_{h \text {,max }}$ defines the phase angle range of harmonic currents. The values can be assumed according to some field measurement research about harmonics [34].

\subsection{Measurement}

\subsubsection{Measurement Points and Quantities}

It is almost impossible to install the measurement units at every $\mathrm{PoC}$ in the distribution network due to the huge economic cost and information capacity. So some points need to be selected as the measurement points and the harmonic states on the non-monitored nodes need to be estimated. In this paper, the measurement points are recommended at the following locations

- At the secondary side of $M V / L V$ transformer (At the beginning point of the feeder).

- At the node and branch of the PoC connected at the end of the feeder.

- At the nodes and branches of PoCs which are likely to produce high level of harmonic currents.

The number of the dimensions for measurement vector $\boldsymbol{M}^{\prime}$ is $11 \times N_{\text {mea }} \times N_{t}$, where $N_{\text {mea }}$ is the number of measurement points in the grid and $N_{t}$ is the number of time steps.

$$
\begin{aligned}
\boldsymbol{M}^{\prime}= & {\left[\boldsymbol{U}_{\boldsymbol{h}}^{\prime}(a, b, c)_{t 1}, \boldsymbol{I}_{\boldsymbol{h}}^{\prime}(a, b, c)_{t 1}, \boldsymbol{\varphi}_{\boldsymbol{h}}^{\prime}(U a, U b, U c)_{t 1}\right.} \\
& \boldsymbol{\varphi}_{\boldsymbol{h}}^{\prime}(I a, I b, I c)_{t 1}, \boldsymbol{U}_{\boldsymbol{h}}^{\prime}(a, b, c)_{t 2}, \boldsymbol{I}_{\boldsymbol{h}}^{\prime}(a, b, c)_{t 2} \\
& \boldsymbol{\varphi}_{\boldsymbol{h}}^{\prime}(U a, U b, U c)_{t 2}, \boldsymbol{\varphi}_{\boldsymbol{h}}^{\prime}(I a, I b, I c)_{t 2}, \ldots, \boldsymbol{U}_{\boldsymbol{h}}^{\prime}(a, b, c)_{t n} \\
& \left.\boldsymbol{I}_{\boldsymbol{h}}^{\prime}(a, b, c)_{t n}, \boldsymbol{\varphi}_{\boldsymbol{h}}^{\prime}(U a, U b, U c)_{t n}, \boldsymbol{\varphi}_{\boldsymbol{h}}^{\prime}(I a, I b, I c)_{t n}\right]
\end{aligned}
$$

where $\boldsymbol{U}_{\boldsymbol{h}}^{\prime}(a, b, c)$ and $\boldsymbol{I}_{\boldsymbol{h}}^{\prime}(a, b, c)$ are the magnitudes of voltages and currents, $\boldsymbol{\varphi}_{\boldsymbol{h}}^{\prime}(U a, U b, U c), \boldsymbol{\varphi}_{\boldsymbol{h}}^{\prime}(I a, I b, I c)$ are the phase angle vectors and $t 1$ to $t h$ represents the time vector since the harmonic pollution is a continuous power quality phenomenon. The phase angle of $h$ order is calculated when the fundamental of phase a is considered as the reference phase. 


\subsubsection{Measurement Error}

The error of measurement devices is generally considered to be normally distributed [35]. So the real value vector is defined as $\boldsymbol{M}$ correspondingly. Its standard deviation $\theta$ can be calculated as:

$$
\theta=\frac{1}{3} \Psi \tau
$$

where $\Psi$ is the measuring range and $\tau$ is the maximum relative error. The absolute error is obviously lower when the measuring range is narrower and at the same time the range should also cover the largest measured value with sufficient margin. For the phase angle measurement, the standard deviation $\theta_{\varphi}$ is:

$$
\theta_{\varphi}=\frac{1}{3} \tau_{\varphi}
$$

where $\tau_{\varphi}$ is the maximum error of phase angle measurement.

\subsubsection{Conditional Distribution of Measured Value}

Given $L$ and $\boldsymbol{G}_{c}$, the real values of measurement vector $\boldsymbol{M}$ can be calculated through harmonic load flow, which can be shown as:

$$
\boldsymbol{M}=h_{l f}\left(L, \boldsymbol{G}_{c}\right)
$$

With a specific disturbance location $l$, grid condition $\boldsymbol{g}_{c}$ and the real value vector of measurement vector $\boldsymbol{m}$, the conditional PDF of $\boldsymbol{M}^{\prime}$ can be calculated as

$$
f_{M^{\prime}}\left(\boldsymbol{m}^{\prime} \mid l, \boldsymbol{g}_{c}\right)=\mathfrak{N}\left(\boldsymbol{m}^{\prime} \mid \boldsymbol{m}, \boldsymbol{\Sigma}_{\boldsymbol{e}}\right)
$$

where $\mathfrak{N}(\cdot \mid \boldsymbol{\mu}, \boldsymbol{\Sigma})$ is the PDF of multivariate normal distribution with mean $\boldsymbol{\mu}$ and covariance matrix $\Sigma, \Sigma_{\boldsymbol{e}}$ is the covariance matrix with measurement uncertainties.

\section{Posterior Distribution of Harmonic Disturbance and Ranking of Suspect- ed Disturbance Locations}

The relevant measurement vector $\boldsymbol{m}^{\text {** }}$ can be obtained with a particular grid condition. Given the harmonic disturbance location $l$, the probability density of $\boldsymbol{m}^{* *}$ can be calculated via the integral on the sample space of grid conditions to consider the different grid conditions, shown in (17)

$$
f_{M^{\prime}}\left(\boldsymbol{m}^{\prime *} \mid l\right)=\int_{\Omega_{G_{c}}} f_{M^{\prime}}\left(\boldsymbol{m}^{\prime *} \mid l, \boldsymbol{g}_{c}\right) f_{\boldsymbol{G}_{c}}\left(\boldsymbol{g}_{c}\right) d \boldsymbol{g}_{c}
$$

Applying the law of total probability, the marginal probability density of $\boldsymbol{m}^{*}$ includes two summations which are corresponding to $f_{L, G D}(l)$ and $f_{L, L D}(l)$ as shown in (18). 


$$
f_{\boldsymbol{M}^{\prime}}\left(\boldsymbol{m}^{\prime *}\right)=\sum_{l \in S_{G D} \cup S_{L D}} f_{\boldsymbol{M}^{\prime}}\left(\boldsymbol{m}^{\prime *} \mid l\right) f_{L, D}(l)
$$

Applying (1), the posterior conditional PMF of disturbance location $L$ given the measurement vector $\boldsymbol{m}^{\prime *}$ can be acquired by (19).

$$
f_{L, D}\left(l \mid \boldsymbol{m}^{* *}\right)=\frac{f_{M^{\prime}}\left(\boldsymbol{m}^{\prime *} \mid l\right) f_{L, D}(l)}{f_{\boldsymbol{M}^{\prime}}\left(\boldsymbol{m}^{\prime *}\right)}
$$

With the posterior distribution of harmonic location, the probability that the harmonic disturbance is from the grid or each particular load can be calculated. Then the ranking of suspected harmonic disturbance location is obtained.

$$
\begin{gathered}
P_{g d}=f_{L, D}\left(0 \mid \boldsymbol{m}^{\prime *}\right) \\
P_{i}=f_{L, D}\left(i \mid \boldsymbol{m}^{\prime *}\right)
\end{gathered}
$$

where $P_{g d}$ is the probability that the harmonic disturbance is from the grid and $P_{i}$ is that from the ith customer.

\section{Monte Carlo Integration with Independent Importance Sampling}

Given the measurement vector $\boldsymbol{m}^{* *}$, for each particular $l$ of the harmonic disturbance location $L, f_{\boldsymbol{M}^{\prime}}\left(\boldsymbol{m}^{\prime *} \mid l\right)$ in (17) needs to be calculated while the number of dimensions of the integral equals to that of the grid condition $\boldsymbol{G}_{c}$, which is $2+2 N_{\text {load }}+2 N_{\text {load }}$. Applying deterministic approach to calculate this high-dimensional integral is almost impossible. Thus, Monte Carlo integration should be applied with independent importance sampling [36], [37]. Since the parameters in $\boldsymbol{G}_{c}$ are not dependent on each other, it is viable to sample each component independently and merge them together. The uniform distribution and truncated normal distribution are carried out in this paper to acquire the $\operatorname{PDF} p\left(\boldsymbol{g}_{c}\right)$.

When $N_{M C}$ random grid conditions are sampled like (22), $f_{\boldsymbol{M}^{\prime}}\left(\boldsymbol{m}^{\prime *} \mid l\right)$ in (17) can be calculated with (23) approximately and the error of the approximation decreases as $1 / \sqrt{N_{M C}}$ theoretically.

$$
\begin{gathered}
p\left(\boldsymbol{g}_{c}\right) \rightarrow \boldsymbol{g}_{c}^{1}, \cdots, \boldsymbol{g}_{c}^{N_{M C}} \in \Omega_{\boldsymbol{G}_{c}} \\
f_{\boldsymbol{M}^{\prime}}\left(\boldsymbol{m}^{\prime *} \mid l^{*}\right) \approx \frac{1}{N_{M C}} \sum_{k=1}^{N_{M C}} \frac{\left.f_{\boldsymbol{M}^{\prime}}\left(\boldsymbol{m}^{\prime *} \mid l^{*}, \boldsymbol{g}_{c}^{k}\right) f_{\boldsymbol{G}_{c}}\left(\boldsymbol{g}_{c}^{k}\right)\right)}{p\left(\boldsymbol{g}_{c}^{k}\right)} \\
=\frac{1}{N_{M C}} \sum_{k=1}^{N_{M C}} f_{\boldsymbol{M}^{\prime}}\left(\boldsymbol{m}^{\prime *} \mid l^{*}, \boldsymbol{g}_{c}^{k}\right)
\end{gathered}
$$




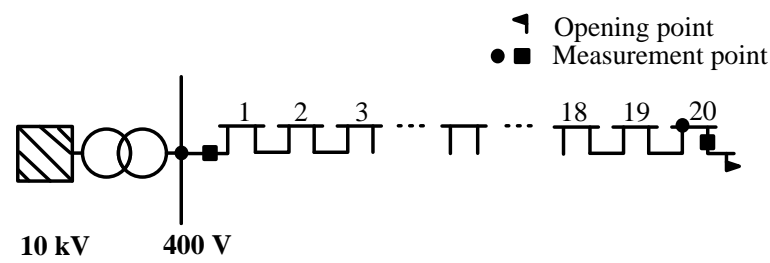

Figure 3: The LV network for case study

\section{Network Modelling and Simulation Algorithm}

In this section, a LV network is modelled and several scenarios are designed to investigate the performance of proposed methodology by simulation. The sensitivity study is carried out with different simulation parameters.

\subsection{Network Modelling}

The LV distribution network in the Netherlands are mostly operated in a radial structure. Fig. 3 shows the topology of LV grid for case study. The feeder is distributed with a $10 / 0.4 \mathrm{kV}$ substation and the cable is made of aluminium with the cross-section of $150 \mathrm{~mm}^{2}$. The other parameters are presented below and some of them will be changed in the simulation to investigate the sensitivity.

- $Z_{e g, \mu}=U_{n}^{2} / S_{s c}$, is the mean value of the normal distribution. $U_{n}=400 \mathrm{~V}$ is the nominal voltage of the grid and $S_{s c}=100 M V A$ is the applied short-circuit power. $Z_{e g, \delta}=0.0001 \Omega$ is the assumed standard deviation when $Z_{e g, \mu}$ is calculated as $0.0016 \Omega$.

- $N_{l}=60, N_{l}$ is the number of PoCs on feeder. In LV network, the customers are usually single-phase connected. So the harmonic load flow is calculated on one phase $N_{\text {load }}$ which has 20 PoCs .

- $h=5 t h$, the investigated harmonic order is $5 t h$.

- In each simulation case, the network state is randomly generated first. Then the real load status can be obtained based on the truncated normal distribution with the covariance matrix $\Sigma_{s e}$. The range of estimated active power is considered same for all loads which is $\mathcal{U}(400 W, 1100 W)$ and $P l_{\delta, i}$ is equal for each load as $30 \mathrm{~W}$. The power factor of the estimated loads are randomly chosen from 0.98 capacitive to 0.95 inductive with $P f_{\delta, i}$ of 0.05 for all loads.

- $U_{s h, p \min }$ and $U_{s h, p \max }$ give the range of background harmonic voltage distortion. The 5 th harmonic voltage distortion is between $2 \%$ to $4.4 \%$ of nominal voltage in one day as shown in [32]. In a relatively short measurement time like 1 hour, the average value of differences between the maximum and minimum distortion in each hour is about $0.2 \%$. Therefore, $U_{s h, p \min }=3 \%$ and $U_{s h, p \max }=3.2 \%$ are assumed. If the disturbance is from the grid side, $U s h p_{\min }$ and $U_{s h, p \max }$ are $3.5 \%$ and $3.7 \%$ respectively. 
- $I_{h, p \min }=5 \%$ and $I_{h, p \max }=8 \%$ give the low and high levels of harmonic current in percentage of the fundamental current. If the disturbance is from one specific customer, $I_{d h, \text { pmin }}$ and $I_{d h, \text { pmax }}$ are $50 \%$ and $80 \%$ when the disturbance level (DL) is 10 by default.

- $\Theta_{h, \min }=270^{\circ}$ and $\Theta_{h, \max }=360^{\circ}$. The phase angle range of $5 t h$ harmonic current is based on the results of field measurement in one LV network in the Netherlands [34].

- The lower and upper bounds of the measuring ranges are shown as $\Psi_{U}$ for voltage, $\Psi_{I}$ for current. The measurement error of phase angle is shown as $\tau_{\varphi} . \Psi_{U} \sim$ $\left[10 \mathrm{~V}, 1.25 U_{n}\right], \Psi_{I} \sim[5 \mathrm{~A}, 200 \mathrm{~A}]$ and $\tau_{\varphi} \leq 5^{\circ}$, where $U_{n}$ is the nominal bus voltage.

- $\theta_{I}=0.3 \mathrm{~A}$ and $\theta_{U}=0.05 \mathrm{~A}$. The sensitivity of measurement accuracy to detection results is investigated in the following section.

- $N_{m}=2$. The initial measurement points are installed at the beginning and and the end of the feeder.

- $N_{t}=6$. The measurement is carried out during the period $T$. The default number of groups of measurement results are assumed to be 6 .

- $N_{M C}=10000$ and $N_{M C}^{\prime}=100$ for the Monte Carlo integration and Monte Carlo simulation.

\subsection{Simulation Algorithm}

Fig. 4 shows the algorithm flow chart of the HDL by simulation. A random grid condition is generated, and the measurement values with error are obtained. Then the probability that the harmonic disturbance is from the grid or each customer is calculated with (20) and (21) applying Monte Carlo integration. In order to get a statistic result, Monte Carlo simulation is run to obtain more cases. Eventually, the average value of the rankings of the real locations in the calculated PMFs is obtained as (24).

$$
\epsilon_{r, a}=\frac{1}{N_{M C}^{\prime}} \sum_{k=1}^{N_{M C}^{\prime}} \epsilon_{k}
$$

where $N_{M C}^{\prime}$ is the number of Monte Carlo simulations and $\epsilon_{k}$ is the ranking of the real location in the calculated PMF. Fig. 5 shows a bar chart of probability of the suspected harmonic source. The 0 on horizontal axis represents the grid side and numbers 1 to 20 represents each customer. In this example, the real disturbance location is at PoC. 8 and the calculated probability at PoC. 8 ranks at the second place. So $\epsilon_{k}$ is 2 in this case and $\epsilon_{r, a}$ can be obtained with $N_{M C}^{\prime}$ repetitions of the simulation. 


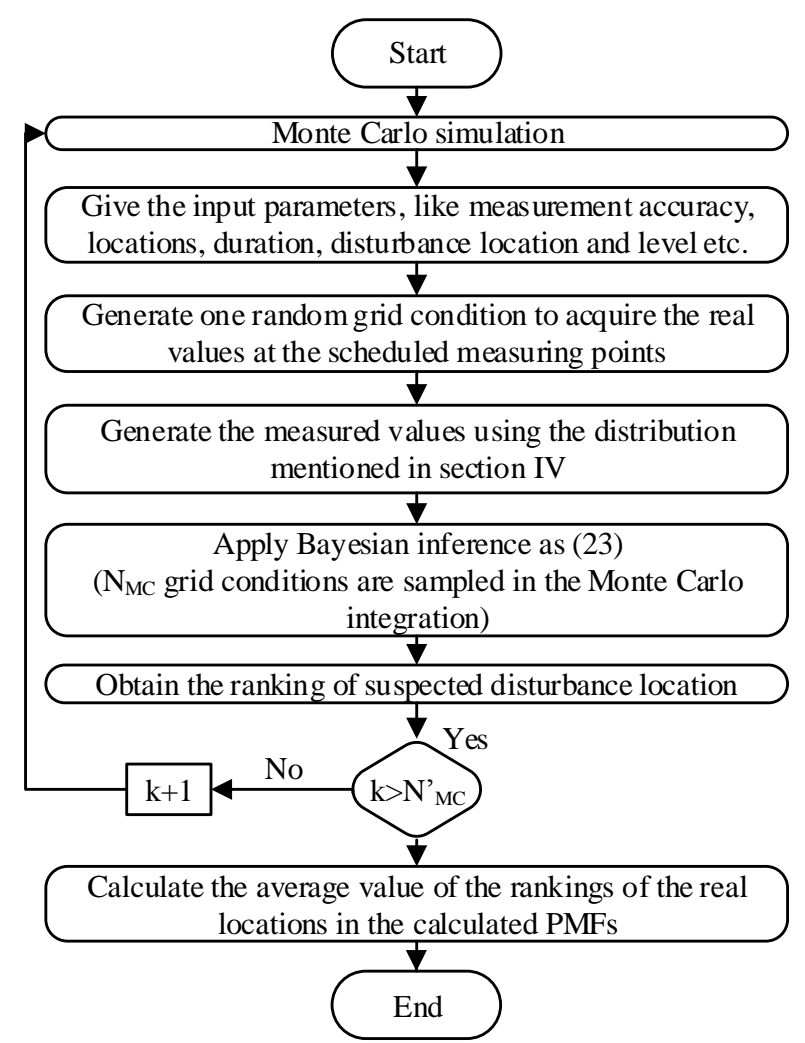

Figure 4: The algorithm flow chart for the simulation

\section{Analysis of Simulation Results}

The sensitivity study of different parameters to the detection performance is carried out in this section. The results can be used to determine the necessary measurement conditions according to the required performance level, or help to know the optimum number of measurements which both considers the performance and the cost.

\subsection{Influence of the Number of Measurement Points, Locations and Measurement Quantities on Time Series}

The detection performance when different number of measurement points are applied is investigated in this section. $\epsilon_{r, a}$ is used as an index to observe the performance

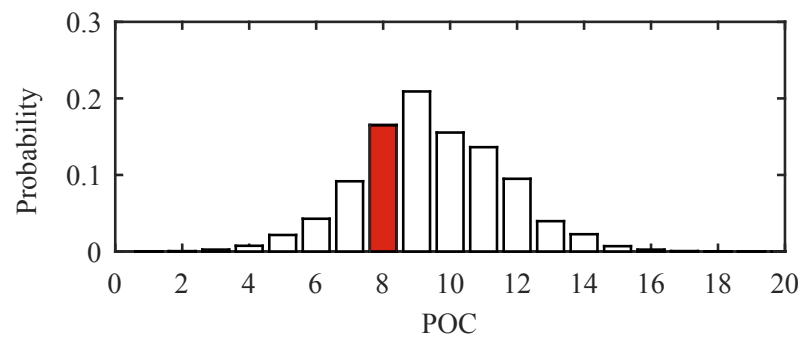

Figure 5: The probability of each location to be the harmonic source 
as shown in Fig. 6. The detection performance is considered better when the index is lower. For 1 measurement point case, it is located at the LV busbar of the transformer while the ending point is also measured when there are two measurement points. The other measurement points are assumed to be evenly installed along the feeder when there are 3 measurement points or more. The location accuracy is found to grow when more measurement devices are installed in the feeder. For example, $\epsilon_{r, a}$ is up to 2 when there are 6 devices installed. In order to investigate the impact of measurement locations, the measurement devices are assumed to be at particular points, which may be feasible when some customers are more likely to produce the disturbance. In the simulation, if there are 3 measurement points on the feeder, besides the beginning and ending points, the third point is assumed at the point of disturbance. The branch current in front of the disturbance source and the voltage at this PoC are measured. If there are 4 measurement devices, besides the previous mentioned 3 points, the current in the branch behind the disturbance source and the voltage at the next PoC are also measured. The comparison with Fig. 6 in which the measurement points are distributed evenly on the feeder are shown in Table 1. The statistics show that the performance is better when the measurement units are installed at the particular points than when the devices are installed evenly on the feeder. Therefore, the additional measuring points can be installed at points which has higher probability to inject disturbance. Since the additional measuring point at ending point brings higher accuracy comparing with the 1 measurement point case, 2-points measurement is assumed in the following parts which discuss the impacts of other parameters. The default number of measurement vectors on time period $T$ is 6 which is considered as 10-min value in 1 hour. Fig. 7 shows the values of $\epsilon_{r, a}$ when $N_{t}$ is $3,6,12,15,20,30,60$ and 120 which represent 20,10, $5,4,3,2,1$ and 0.5 -min measurement values respectively. It shows more measurement quantities which bring more information increases the detection accuracy.

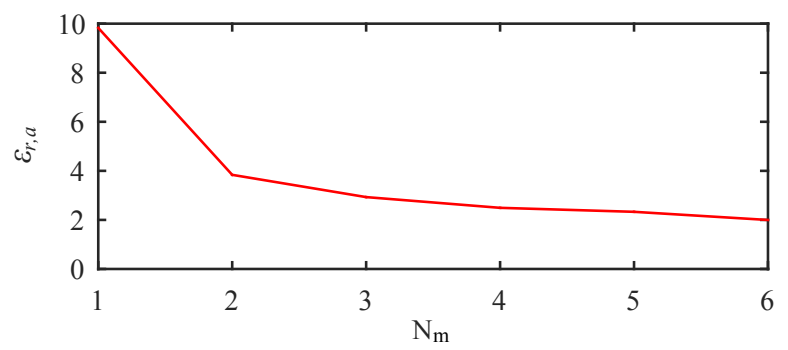

Figure 6: The values of $\epsilon_{r, a}$ with different number of measurement points

\subsection{Influence of Disturbance Level}

In this part, the different disturbance levels of loads are applied and Fig. 8 shows the values of $\epsilon_{r, a}$ with different disturbance levels. The disturbance level means the integer multiples of the injection value from non-disturbing customer. It is found that the detection accuracy is better when the disturbance level is higher. The mean of indices is lower than 3 when the disturbance level is more than 15, which is considered as a good performance. 


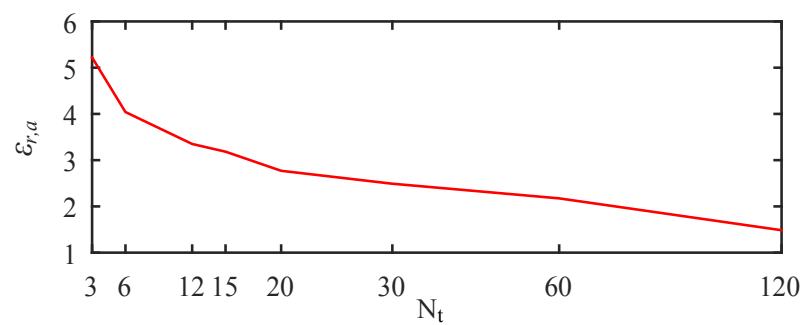

Figure 7: The values of $\epsilon_{r, a}$ with different measurement time steps

Table 1: The comparison between the cases when the measurement locations are evenly distributed and at the particular points

\begin{tabular}{|c|c|c|}
\hline Cases of locations & case 1 & case 2 \\
\hline Number of points & 2.930 & 2.258 \\
\hline 3 points & 2.493 & 1.441 \\
\hline
\end{tabular}

case1, the measurement points are evenly distributed;

case2: the measurement locations are at particular points

\subsection{Influence of Measurement Parameters and Accuracy}

One important advantage of applying Bayesian inference is to consider the measurement error of devices. Fig. 9 shows the influence of different measurement accuracy levels of both current and voltage to the detection accuracy. Fig. 9(a) shows the values of $\epsilon_{r, a}$ changes from 3.8 to 4.5 when $\theta_{I}$ increases from $0.1 A$ to $0.8 A\left(\theta_{U}=0.03 \mathrm{~V}\right)$. Generally, the value of $\epsilon_{r, a}$ is higher when the current measurement accuracy level of device is lower. However, it is not a continuous growth because the detection result is sensitive to other parameters applied in Monte Carlo simulation as well. A smooth growing curve can be obtained if a larger number of Monte Carlo simulations is run which can be very time-consuming. Comparing with the results shown in Fig. 9(a), the detection performance shows to be more sensitive to the accuracy level of voltage measurement accuracy (Fig. 9(b)). The value increases from 2.6 to 8.2 when the accuracy level of voltage measurement decreases $\left(\theta_{U}\right.$ grows from $0.01 \mathrm{~V}$ to $0.19 \mathrm{~V}$ and $\left.\theta_{I}=0.3 \mathrm{~A}\right)$. The sensitivity of phase angle to the detection result is not significant while the total change of $\epsilon_{r, a}$ is about 0.8 with different measurement accuracy level of phase angle $\left(\theta_{\varphi}=5^{\circ}\right.$ to $40^{\circ}$ ). Fortunately, the voltage measurement accuracy in LV grid can be quite high since the voltage signal can be sampled directly while the current measurement may have relatively bigger error because current transformers (clamp) are mostly applied.

\subsection{Influence of the Definition of Prior Distribution}

In the previous simulation, the marginal probability as shown in (3) of grid and each customer is considered equally. However in reality, some customers may have a higher probability to be the harmonic pollution source than others because of particular 


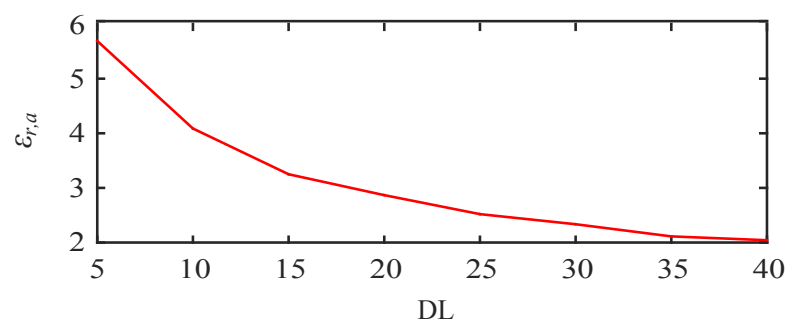

Figure 8: The values of $\epsilon_{r, a}$ with different disturbance levels
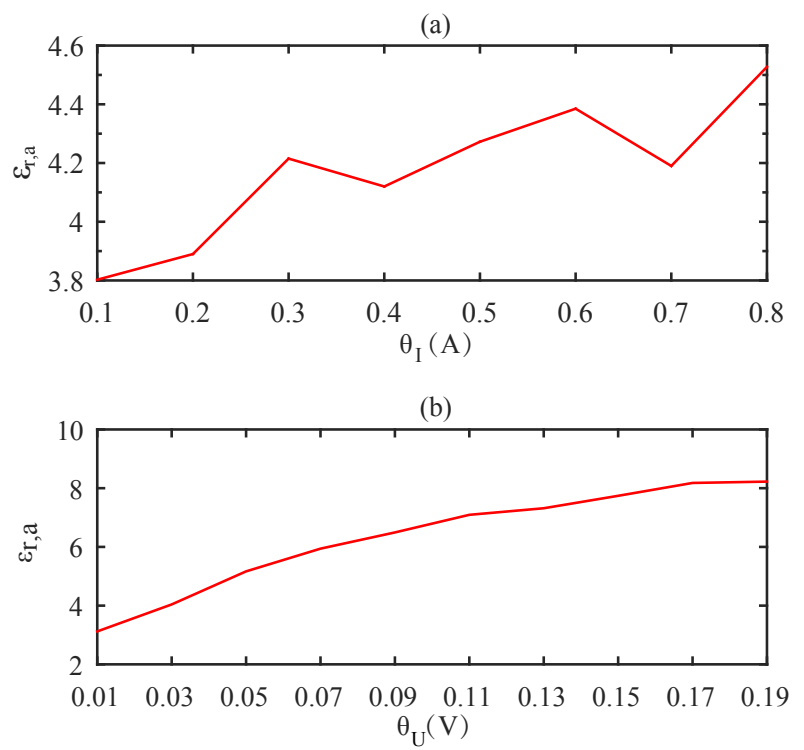

Figure 9: The values of $\epsilon_{r, a}$ with different measurement accuracy levels (a) Current. (b) Voltage.

combination of loads and the prior distribution can be modified to get a more accurate estimation. In this section, the marginal probability to be the harmonic source of one particular customer is assumed to be $m \%$ more than other customers as shown in (25). As shown in the Fig. $10, m$ is considered to be from $0 \%$ to $100 \%$ which means the marginal probability of the particular customer is 1 to 2 times as other customers. It is clear that more accurate definition of prior distribution will bring better estimation.

$$
f_{L, D}(l)=\left\{\begin{array}{cl}
P_{G D} & l \in S_{G D} \\
\frac{(1+m) P_{L D}}{\left(1+m+N_{\text {load }}\right)} & l \in S_{i t h} \\
\frac{P_{L D}}{\left(1+m+N_{\text {load }}\right)} & l \in S_{L D 1}
\end{array}\right.
$$

where $S_{i t h}$ is the particular customer which has a higher probability to inject harmonic and $S_{L D 1}$ is the set of the other customers. 


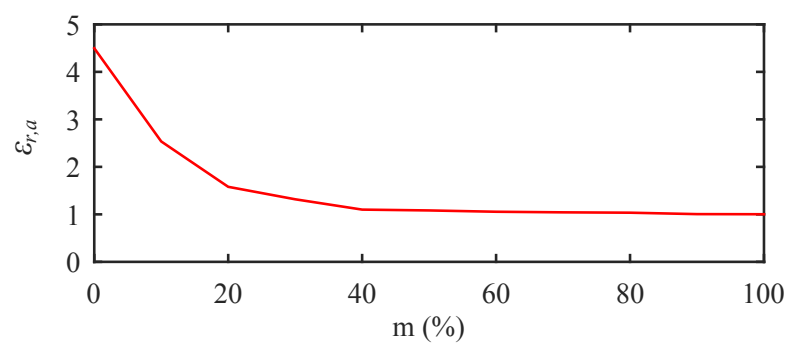

Figure 10: The values of $\epsilon_{r, a}$ with different prior distributions

\subsection{Summary}

Table 2 shows the approximate required value of each individual parameter to achieve a certain performance level $\left(\epsilon_{r, a}\right)$ when the other parameters are kept at their default settings. For the cases that the disturbance level is not remarkably high, better results can be achieved with more measurement data or higher measurement accuracy. However, the measurement accuracy level of current is less influential than that of voltage. If more research on the priori has been done, a non-uniform prior distribution can be applied to have a better performance. In addition, the combined adjustment of several measurement parameters could help to achieve the required performance. For example, when the second row of Table 2 which are $N_{m}=3, N_{t}=15, D L=15$, $\theta_{I}=0.3 \mathrm{~A}, \theta_{U}=0.01 \mathrm{~V}$ and $m=7$ are applied in the simulation simultaneously, the value of $\epsilon_{r, a}$ almost equals to 1 instead of 3 which is achieved by the adjustment of single parameter.

Table 2: The approximate required value of each individual parameter to achieve a certain $\epsilon_{r, a}$ when the other parameters are kept at their default settings

\begin{tabular}{|c|c|c|c|c|c|c|c|c|c|c|c|}
\hline$\epsilon_{r, a}$ & $N_{m}$ & $\epsilon_{r, a}$ & $N_{t}$ & $\epsilon_{r, a}$ & DL & $\epsilon_{r, a}$ & $\theta_{I}(I)$ & $\epsilon_{r, a}$ & $\theta_{U}(V)$ & $\epsilon_{r, a}$ & $m(\%)$ \\
\hline 2 & 5 & 2 & 60 & 2 & 40 & 2 & - & 2 & - & 2 & 15 \\
\hline 3 & 3 & 3 & 15 & 3 & 15 & 3 & - & 3 & 0.01 & 3 & 7 \\
\hline 4 & 2 & 4 & 6 & 4 & 10 & 4 & 0.1 & 4 & 0.03 & 4 & 0 \\
\hline 5 & - & 5 & 3 & 5 & 5 & 5 & 0.8 & 5 & 0.05 & 5 & - \\
\hline
\end{tabular}

- means the value is not available from the results

\subsection{Comparison with other Methods}

Previously, the main three strategies applied to detect the harmonic source were based on the harmonic power direction [1], [3], [4], [5], [6], [7], the critical impedance [14], [15], [16] and the harmonic state estimation (HSE) [18], [19], [20] respectively. Applying the harmonic power direction method, the harmonic source is determined to be either upstream or downstream of the metering section can be determined by the positive or negative sign of the calculated harmonic power. The principle of critical impedance method is to compare the harmonic voltage magnitudes in the Thevenin equivalent circuits of grid and load sides and to choose the larger one as the main 
harmonic source. Since operators can only know which side of the measurement node has the harmonic source, these two methods are applicable only when a particular load is suspected to be the disturbance source and it is measured at this particular PoC. If the whole network needs to be investigated, the measurement meters are required at all nodes of the power system. Therefore, they are considered as single point measurement methods (SPMM). In addition, uncertainties are hardly to be taken into account if either of them is used. To compare the performance of the SPMM and the proposed method based on Bayesian inference (BIM), the measurement condition (the number of devices etc.) for applying two methods is assumed to be same. A new index is defined as (26) for BIM.

$$
\epsilon_{p, a}=\frac{1}{N_{M C}^{\prime}} \sum_{k=1}^{N_{M C}^{\prime}} \epsilon_{p, k}
$$

where $\epsilon_{p, k}$ is the probability of the real location in the calculated PMF at the $k t h$ Monte Carlo simulation and $\epsilon_{p, a}$ is the average probability of $N_{M C}^{\prime}$ times Monte Carlo simulations. $\epsilon_{p, a}$ could be also concerned as the probability of detection accuracy. For the case in Fig. $5, \epsilon_{p, k}$ at POC. 8 is about 0.18. The performance is considered better when $\epsilon_{p, a}$ is higher. For SPMM, the probability of detection accuracy could be calculated analytically. Since one measurement device is supposed to be installed at the beginning point of the feeder, the conditional probability of detection accuracy is 1 if the harmonic disturbance is at the external grid $\left(P_{c o n, G D}\right)$ when the measurement error is ignored. If the harmonic disturbance is at the load side, the conditional probability of detection accuracy $\left(P_{c o n, L D, i}\right)$ is dependent on the number of measurement installations. For the case of one measurement device, $P_{c o n, L D, i}$ is $1 / 20$ because 20 loads are connected in the system.

$$
\begin{gathered}
P_{\text {con }, G D}=1 \\
P_{\text {con }, L D, i}=N_{m} / N_{\text {load }}
\end{gathered}
$$

where the measurement devices are assumed to be installed evenly on the feeder. Together with (3), the total probability of detection accuracy for SPMM can be calculated. Fig. 11 shows the values of $\epsilon_{p, a}$ with different number of measurements and it is found BIM shows higher values of $\epsilon_{p, a}$ when there are more than 1 measurement points even with the consideration of uncertainties. Moreover, it should be noted that the best scenario to apply SPMM is taken into consideration when the probability of detection accuracy is calculated. In other words, the disturbance location (either side of the meter) identified by SPMM is assumed to be always accurate which may be too optimistic for the practical application [38].

HSE method could achieve the goal with measurements on selected buses. However, a minimum amount of independent measurements for voltage, real and reactive power which is $2 N_{\text {node }}-1$ is still required to ensure the observability of the system $\left(N_{\text {node }}\right.$ is the number of nodes), otherwise the state estimation problem cannot be solved [27]. 


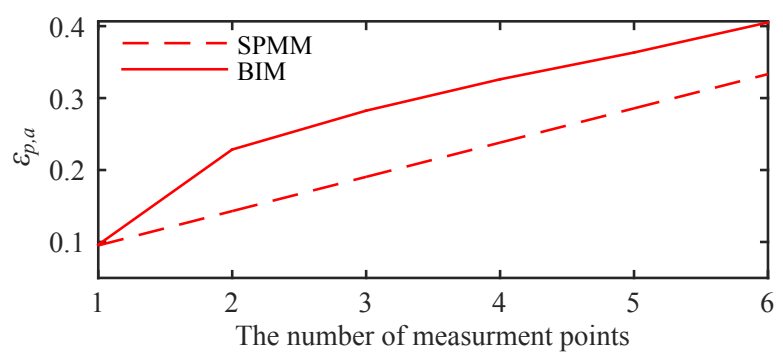

Figure 11: The values of $\epsilon_{p, a}$ with number of different measurement points

Comparing with that, the Bayesian inference has great flexibility to define the measurement scalar or vector which is clearly shown in the paper. The main advantage of the proposed method is to give results of HDL with an indication of their reliability when uncertainties are concerned.

\section{Conclusion}

In this paper, a new method for harmonic disturbance location in distribution network, which is mainly based on Bayesian inference, is proposed. Bayesian inference is applied in order to consider the uncertainties of measurement values and the dynamic states of grid (load distribution, etc.). The conditional probability expressed as the ranking of suspected disturbance locations are obtained based on the prior distribution (grid conditions) and measurement data. It can be used as a tool to locate the harmonic disturbance with limited measurement information in the future.

Monte Carlo simulation is carried out to obtain the statistic results. It shows the proposed method performs quite well when the accuracy level of measurement device is higher than a certain level. And generally, the performance is proportional to the measurement accuracy level. The detection results are more accurate when the harmonic pollution is higher. On the other hand, more measurement points will bring better performance. If the measurement locations can be decided according to the known information, for example, installing at the $\mathrm{PoC}$ which is more likely to inject higher harmonic pollution, then the detection is more effective.

It is essential for the estimation results to choose proper prior. An unrealistic definition of the prior will bring results with large error. Thus the most concerned disadvantage is that it requires a large amount of efforts to get a proper assumption. Another disadvantage may need to be concerned is that the computation time and the needed CPU power for applying Bayesian inference are generally bigger.

\section{References}

[1] D. Saxena, S. Bhaumik, S. Singh, Identification of multiple harmonic sources in power system using optimally placed voltage measurement devices, IEEE Transactions on Industrial Electronics 61 (5) (2014) 2483-2492. 
[2] M. Najjar, G. Heydt, A hybrid nonlinear-least squares estimation of harmonic signal levels in power systems, IEEE Transactions on Power Delivery 6 (1) (1991) $282-288$.

[3] T. Tanaka, H. Akagi, A new method of harmonic power detection based on the instantaneous active power in three-phase circuits, IEEE Transactions on Power Delivery 10 (4) (1995) 1737-1742.

[4] L. Cristaldi, A. Ferrero, Harmonic power flow analysis for the measurement of the electric power quality, IEEE Transactions on Instrumentation and Measurement 44 (3) (1995) 683-685.

[5] W. A. Omran, H. S. K. El-Goharey, M. Kazerani, M. M. A. Salama, Identification and measurement of harmonic pollution for radial and nonradial systems, IEEE Transactions on Power Delivery 24 (3) (2009) 1642-1650.

[6] A. Cataliotti, V. Cosentino, A new measurement method for the detection of harmonic sources in power systems based on the approach of the ieee std. 1459 -2000, IEEE Transactions on Power Delivery 25 (1) (2010) 332-340.

[7] S. Nath, P. Sinha, S. K. Goswami, A wavelet based novel method for the detection of harmonic sources in power systems, International Journal of Electrical Power \& Energy Systems 40 (1) (2012) $54-61$.

[8] J. Farach, W. Grady, A. Arapostathis, An optimal procedure for placing sensors and estimating the locations of harmonic sources in power systems, IEEE Transactions on Power Delivery 8 (3) (1993) 1303-1310.

[9] E. Thunberg, L. Soder, A norton approach to distribution network modeling for harmonic studies, IEEE Transactions on Power Delivery 14 (1) (1999) 272-277.

[10] A. de Oliveira, J. de Oliveira, J. Resende, M. Miskulin, Practical approaches for ac system harmonic impedance measurements, IEEE Transactions on Power Delivery 6 (4) (1991) 1721-1726.

[11] M. Tsukamoto, S. Ogawa, Y. Natsuda, Y. Minowa, S. Nishimura, Advanced technology to identify harmonics characteristics and results of measuring, in: 9th International Conference on Harmonics and Quality of Power (ICHQP), Vol. 1, 2000, pp. 341-346 vol.1.

[12] T. Pyzalski, R. Lukomski, K. Wilkosz, Utilization of the voltage rate to localization of harmonic sources in a power system, in: 12th IEEE Mediterranean Electrotechnical Conference (MELECON), Vol. 3, 2004, pp. 1091-1094 Vol.3.

[13] H. Ma, A. Girgis, Identification and tracking of harmonic sources in a power system using a kalman filter, IEEE Transactions on Power Delivery 11 (3) (1996) 16591665. 
[14] C. Chen, X. Liu, D. Koval, W. Xu, T. Tayjasanant, Critical impedance method a new detecting harmonic sources method in distribution systems, IEEE Transactions on Power Delivery 19 (1) (2004) 288-297.

[15] C. Li, W. Xu, T. Tayjasanant, A "critical impedance"-based method for identifying harmonic sources, IEEE Transactions on Power Delivery 19 (2) (2004) 671-678.

[16] M. Moradloo, M. Tabrizi, H. Karshenas, A new method for identification of main harmonic source based on the superposition and critical impedance methods, in: 40th North American Power Symposium (NAPS), 2008, pp. 1-6.

[17] A. M. Dan, Identification of individual harmonic sources and evaluation their contribution in the harmonic distortion level, in: IEEE Power Energy Society General Meeting, 2009, pp. 1-6.

[18] Z.-P. Du, J. Arrillaga, N. Watson, S. Chen, Identification of harmonic sources of power systems using state estimation, IEE Proceedings-Generation, Transmission and Distribution, 146 (1) (1999) 7-12.

[19] B. Kumar, A.and Das, J. Sharma, Determination of location of multiple harmonic sources in a power system, International Journal of Electrical Power \& Energy Systems 26 (2004) 73-78.

[20] G. D'Antona, C. Muscas, S. Sulis, Localization of nonlinear loads in electric systems through harmonic source estimation, IEEE Transactions on Instrumentation and Measurement 60 (10) (2011) 3423-3430.

[21] D. Srinivasan, W. S. Ng, A. C. Liew, Neural-network-based signature recognition for harmonic source identification, IEEE Transactions on Power Delivery 21 (1) (2006) 398-405.

[22] H. C. Lin, Intelligent neural network-based fast power system harmonic detection, IEEE Transactions on Industrial Electronics 54 (1) (2007) 43-52.

[23] H. C. Lin, Power harmonics and interharmonics measurement using recursive group-harmonic power minimizing algorithm, IEEE Transactions on Industrial Electronics 59 (2) (2012) 1184-1193.

[24] S. Nawi, J. Johari, A. Abidin, A fuzzy logic application for identification of harmonics disturbances sources, in: National Power Engineering Conference (PECon), 2003, pp. 27-31.

[25] M. Farhoodnea, A. Mohamed, H. Shareef, A new method for determining multiple harmonic source locations in a power distribution system, in: IEEE International Conference on Power and Energy (PECon), 2010, pp. 146-150. 
[26] J. Yang, Y. Yang, J. Chen, L. Fu, Z. He, Determining the harmonic contributions of multiple harmonic sources using data clustering analysis, in: International Symposium on Smart Electric Distribution Systems and Technologies (EDST), 2015, pp. 405-411.

[27] Y. Xiang, P. Ribeiro, J. Cobben, Optimization of state-estimator-based operation framework including measurement placement for medium voltage distribution grid, IEEE Transactions on Smart Grid PP (99) (2014) 1-1.

[28] B. Carlin, T. Louis, Bayesian Methods for Data Analysis, Third Edition, Chapman \& Hall/CRC Texts in Statistical Science, Taylor \& Francis, 2011.

[29] J. C. C. Dirk P. Kroese, Statistical Modeling and Computation, Springer New York, 2014.

[30] R. Hogg, E. Tanis, D. Zimmerman, Probability and Statistical Inference, Pearson Education, 2014.

[31] S. Bhattacharyya, Power quality requirements and responsibilities at the point of connection, Ph.D. thesis, Eindhoven Univertsity of Technology (2011).

[32] N. of Experts for Standardisation, Power quality in european electricity supply networks- 2nd edition, Tech. rep., Union of the Electricity Industry EURELECTRIC (2003).

[33] L. Berthet, P. Eyrolles, J. Gauthier, S. Sabeg, Harmonic level measurements on french low-voltage networks, in: 19th International Conference on Electricity Distribution, 2007.

[34] V. Cuk, J. Cobben, W. Kling, P. Ribeiro, Analysis of harmonic current summation based on field measurements, IET Generation, Transmission Distribution 7 (12) (2013) 1391-1400.

[35] R. Singh, B. Pal, R. Vinter, Measurement placement in distribution system state estimation, IEEE Transactions on Power Systems 24 (2) (2009) 668-675.

[36] M. Evans, T. Swartz, Approximating Integrals via Monte Carlo and Deterministic Methods, Oxford Statistical Science Series, 2000.

[37] Y. Xiang, C. J.F.G., A bayesian approach for fault location in medium voltage grids with underground cables, IEEE Power and Energy Technology Systems Journal PP (99) (2015) 1-9.

[38] W. Xu, Power direction method cannot be used for harmonic source detection, in: IEEE Power Engineering Society Summer Meeting, Vol. 2, 2000, pp. 873-876 vol. 2. 